PACS: $13.85 . \mathrm{Hd}, 13.85 .-\mathrm{t}, 13.66 . \mathrm{Bc}, 84.35+\mathrm{I}, 07.05 . \mathrm{Tp}$.

\title{
EMPIRICAL EQUATION USING GMDH METHODOLOGY FOR THE CHARGED PARTICLES MULTIPLICITY DISTRIBUTION IN HADRONIC POSITRON-ELECTRON ANNIHILATION
}

\author{
E.A. El-Dahshan ${ }^{1,2}$, S.Y. El-Bakry ${ }^{1}$ \\ ${ }^{1}$ Department of Physics, Faculty of Sciences, Ain Shams University \\ Abbassia, Postal Code: 11566, Cairo, Egypt \\ ${ }^{2}$ Egyptian E-Learning University (EELU) \\ 33 Elmesah Street, Eldoki, Postal Code: 11261, El-Geiza \\ e_eldahshan@yahoo.com,seldahshan@eelu.edu.eg,salahyaseen_elbakry@sci.asu.edu.eg \\ Received April 12, 2017, revised June 7, 2017, accepted August 23, 2017
}

Multiplicity distributions are the most general characteristics of hadronic multiparticle production processes. The multiplicity distribution of hadronic positron-electron annihilation is investigated using group method data handling (GMDH) technique up to the highest available center of mass energy $(\sqrt{s}$ ) (from $14 \mathrm{GeV}$ to $206 \mathrm{GeV}$ ). We have obtained an empirical physical equation for the multiplicity distribution as a function of $\sqrt{s}$ and the charged multiplicity $\left(\mathrm{n}_{\mathrm{ch}}\right)$ i. e. $P\left(n_{c h}, \sqrt{s}\right)$. Based on the obtained equation, we have also calculated the energy dependence of average multiplicity $(\bar{n})$ i.e. $\bar{n}=\bar{n}(\sqrt{s})$. Our results are compared with the available experimental and theoretical values.

KEYWORDS: hadronic positron-electron annihilation, charged-particles multiplicity distribution, empirical modeling, neural networks, group method of data handling (GMDH).

The charged particle multiplicity (the total number of charged particles produced in an event) in positron-electron annihilation into multi-hadron final states is one of the most fundamental observables in the fragmentation process during which quark-antiquark pairs are produced [1-5]. For example, the interactions among the elementary particles are represented by Feynman diagrams such as those in the following Figure [1].

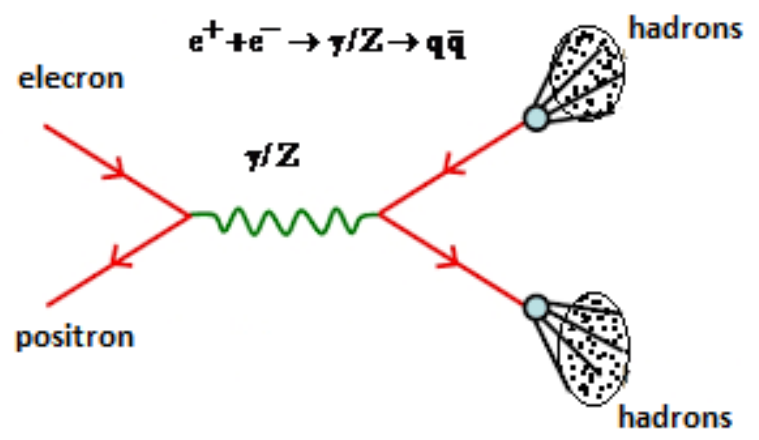

Fig. 1. Feynman diagrams for the annihilation of an electron-positron $\left(e^{+} e^{-}\right)$pair into a virtual $\gamma$ photon or $\mathbf{Z}$ boson and its decay

$$
\text { into a quark-antiquark }(q \bar{q}) \text { pair: } e^{+} e^{-} \rightarrow\left(Z_{o} / \gamma\right) \rightarrow q+\bar{q}[1] .
$$

The $e^{+} e^{-}$annihilation process is well understood by the creation of a quark-antiquark pair, branching of these pairs in accordance to perturbative quantum chromo-dynamics (QCD) and finally hadronization [5]. The analysis and investigation of multiplicity production is the first step for understanding the particle production mechanism, especially in $e^{+} e^{-}$annihilation, it can provide additional information on hadronic final states [6-11]. Multiplicity distributions can be characterized either in terms of probability, $P_{n}$, of producing, $\mathrm{n}_{\mathrm{ch}}$, charged particles at energy ,E, i.e. $P_{n}(E)$, or by the moments of these distributions. The normal method of studying the charged-particle multiplicity distribution and its shape, is to calculate its moments. The common behaviors of the charged-particle multiplicity distribution are obtained using low-order moments, such as the mean, $\bar{n}$, the dispersion, $\mathrm{D}$, which estimates the width of the distribution, the skewness, S, which measures how symmetric the distribution is, and the kurtosis, K, which measures how sharply peaked the distribution is [8]. The multiplicity distribution are treated experimentally (see e.g. [12-20]) and theoretically [21-24]. However, due to the lacking of fundamental theory, a number of phenomenological models have been proposed to characterize the charged-particle multiplicity in high energy hadron processes [1,9], starting with early investigations by W. Heisenberg and E. Fermi [19, 20]. Furthermore, because of the shortage of the fundamental theory to describe the experimental data on multiplicity distribution, the investigation of charged particles production in 
hadronic collisions is still phenomenological and based on a wide class of models and some theoretical principles. We shall study the distribution of the number of charged particles produced in positron-electron hadronic annihilation i. e. the charged multiplicity distribution and the average multiplicity (first order moment). The probability of producing a given number of charged prongs in an inelastic collision can be written as $[1,8]$ :

$$
P=\frac{\sigma_{n}}{\sigma_{\text {inel }}}=\frac{\sigma_{n}}{\sum_{n} \sigma_{n}},
$$

where $\sigma_{n}=$ the topological cross-section for n-prong events, and $\sigma_{\text {inel }}=$ the total inelastic cross-section.

The average charged multiplicity, $\bar{n}$, is defined by [9-11]:

$$
\bar{n}=\sum_{n=1}^{\infty} n P .
$$

Recently, computational intelligence (CI) methodologies have become one of the most efficient techniques for the analysis of the charged particles in positron-electron annihilation (High energy physics "HEP" particle interactions) [25-31]. Generally, CI is a broad term covering a wide range of computational methodologies and approaches (such as artificial neural networks (ANNs), Genetic Programming (GP), .....) and most of them are nature-inspired algorithms. $\mathrm{CI}$ approaches have been used in many fields in particles and nuclear physics as in the other fields, such as studies of event selection problems with Genetic programming (J. M. Link; et.al. 2005) [25], re-discover certain laws of physicssuch as Hamiltonian, Lagrangian, and other laws of geometric and momentum conservation (M. Schmidt, H. Lipson, 2009) [26], estimation of the fission barrier heights of the nuclei (A. Serkan, and T. Bayram, 2014) [27], estimations of beta-decay energies through the Nuclidic Chart (S. Akkoyun et al., 2014) [28] and searching for exotic particles in highenergy physics (P. Baldi et al.2016 and 2014) [ 30,31].

In the light of this, ANNs are considered as nonlinear and highly flexible models that successfully model and analyze any nonlinear problem such as particle physics problems. Given enough data, they can approximate the underlying function for the given problem with high precision [32]. However, the main drawback of ANN approach is concentrated on the neural network architecture. In addition to that, the required time of learning causes difficulties for using ANN in real time system for modeling and regression. Group method data handling (GMDH) type Neural Networks enables restoring the unknown nonlinear regression in parametric form (as an empirical equation). The basic idea of the GMDH is the utilizing of feed-forward networks depending on short-term polynomial transfer functions whose coefficients are obtained utilizing regression combined with the self organizing activity behind neural network [33-36].

The ANN and the GMDH are inductive algorithms able to build non-linear connections between a set of input data and the output, without need for complex theory [36]. The two algorithms (GMDH and ANN) are combined to develop GMDH-Neural Network paradigm. The GMDH can be used for demonstrating and approximating any nonlinear complex system. It has been found that, the GMDH is an accurate simplified model for inaccurate or noisy data sets.

In the present work, we have used the GMDH-neural network (to obtain empirical physical equation) to model and analyze the charged distribution in hadronic positron-electron annihilation at center of mass energy, $\sqrt{s}$, i.e. $P\left(n_{c h}, \sqrt{s}\right)$, ranging from 14 to $206 \mathrm{GeV}$ [12-20] as well as the energy dependence of average multiplicity, $\overline{\mathrm{n}}$. The obtained results are compared with the ones from other models [21-24]. The success of the approach used here is promising for modeling systems for which the relationships between the interaction parameters are not well understood and for which precise data is not available.

The paper is organized as follows: section GROUP METHOD DATA HANDLING (GMDH) gives brief introduction on the GMDH approach. Details of the polynomial model for charged particles multiplicity distribution in hadronic $e^{+} e^{-}$annihilation are described in section PROPOSED MODEL FOR THE CHARGED-PARTICLES MULTIPLICITY DISTRIBUTION IN HADRONIC POSITRON-ELECTRON ANNIHILATION. The results obtained are presented in section RESULTS AND DISCUSSION. Finally, the main conclusions of this study are formulated in section CONCLUSIONS.

\section{GROUP METHOD DATA HANDLING (GMDH)}

One of the potential issues of using ANN based approaches in any domain is the possible choice of different architectures, network types, learning paradigm, layer topologies and sizes. Trials and errors paradigm are often used to choose the type and topology of a network for a given problem, and this can give poor performance. The utilization of GMDH neural networks can guide users with these choices and diminish the requirement for a priori knowledge about the model for the problem to be solved [33-36]. The GMDH is able to extract knowledge about the system under observation directly from data sampling. GMDH was developed by A.G. Ivakhnenko in the end of 1960s for identifying non-linear relation between input and output variables. A.G. Ivakhnenko was inspired by the form of KolmogorovGabor polynomials which is the discrete analogue of Volterra Function series [33-36]. The central idea behind the GMDH technique is that it tries to build a mathematical function (called polynomial model) that behaves as closely as possible to the way the predicted and the actual values of the output would. The GMDH problem consists of 
constructing a polynomial function $\hat{f}$ that could simulate and model the actual one, $f$, in order to predict output $£$ for a given input vector $X=\left(x_{1}, x_{2}, x_{3}, \ldots \ldots x_{n}\right)$; as close as possible to its actual output $\mathrm{y}$. Therefore, given $\mathrm{M}$ observations of "multi-input single-output" pairs, the problem consists of finding $y_{i}$ as follows:

$$
y_{i}=f\left(x_{i 1}, x_{i 2}, x_{i 3}, \ldots \ldots x_{i n}\right) \quad(i=1,2, \ldots . . M)
$$

In the next step, GMDH-type neural network is trained in order to approximate the output values for a given input vector:

$$
\hat{y}=\hat{f}\left(x_{i 1}, x_{i 2}, x_{i 3}, \ldots \ldots x_{i n}\right)(i=1,2, \ldots . M)
$$

The function $\hat{f}$ is determined so that the sum of square difference between predicted values and actual ones is minimized as follows:

$$
\sum_{i=1}^{M}\left[\hat{f}\left(x_{i 1}, x_{i 2,} x_{i 3}, \ldots \ldots x_{i n}\right)-y_{i}\right]^{2} \rightarrow \min
$$

Ivakhnenko employed Kolmorovo-Gaborov sentence [33], which proves that every function $y_{n}=f(x)$ can be represented by an infinite Volterra-Kolmogorov-Gabor (VKG) polynomial [33-36] of the form:

$$
y=f\left(x_{i 1}, x_{i 2}, x_{i 3}, \ldots \ldots, x_{i n}\right)=a_{0}+\sum_{i=1}^{n} a_{i} x_{i}+\sum_{i=1}^{n} \sum_{j=1}^{n} a_{i j} x_{i} x_{j}+\sum_{i=1}^{n} \sum_{j=1}^{n} \sum_{k=1}^{n} a_{i j k} x_{i} x_{j} x_{k}+\ldots \ldots .
$$

where $X=\left(x_{1}, x_{2}, x_{3}, \ldots \ldots x_{M}\right)$ is the vector input variables and $A=\left(a_{i}, a_{i j} a_{i j k}, \ldots ..\right)$ is the vector of coefficients of weights. This mathematical form can be characterized by a system of partial quadratic polynomials (referred to as Partial descriptor "PD") consisting of only two variables (neurons: each neuron is considered as the partial model) as follows:

$$
\hat{y}=G\left(x_{i}, x_{j}\right)=a_{0}+a_{1} x_{i}+a_{2} x_{i}+a_{3} x_{i} x_{j}+a_{4} x_{i}^{2}+a_{5} x_{j}^{2}
$$

Thus, such PD is recursively utilized in the network of connected neuron to establish the general mathematical form of the inputs and outputs variables given in equation (6). The coefficients $a_{i}$ in equation (7) are calculated using regression techniques during the learning process. Also, the GMDH network model is constructed during the learning process based on the experimental data. The experimental data, including inputs "independent variables" $\left(x_{1}, x_{2}, x_{3}, \ldots \ldots x_{n}\right)$ and output (one dependent variable " $y$ ") is split into a training and testing set. During a learning process a forward multi-layer neural network is developed (see Fig. 2). The GMDH network learns in an inductive manner and builds a function (called a polynomial) model) that results in the minimum error between the predicted value and expected output. The resulting network can be represented as a polynomial of polynomial system in the form of explicit mathematical equation. This inductive approach to determining the model structure notably reduces the amount of a priori knowledge required from the user and allows selecting structure that best follows the given dataset. For further details, the authors refer to read [33-36].

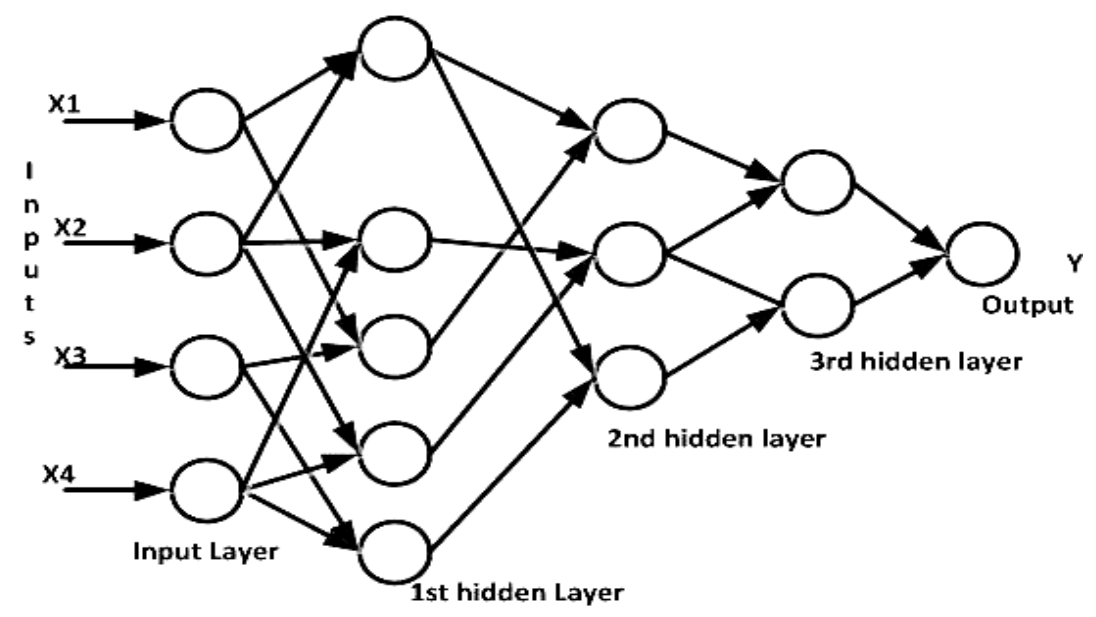

Fig. 2. General structure of GMDH polynomial neural network

\section{PROPOSED MODEL FOR THE CHARGED-PARTICLES MULTIPLICITY DISTRIBUTION IN HADRONIC POSITRON-ELECTRON ANNIHILATION}

The analysis of hadronic positron-electron annihilation founded by a mathematical models based on the traditional methods is complicate. Today, advances in information technology and data analysis approaches have forced the physicists to use the computational intelligence $(\mathrm{CI})$ in modeling and analyzing physical phenomena, especially in high energy physics (HEP). Moreover, computational intelligence methodologies have become one of the most efficient 
techniques for the analysis of the charged particles in positron-electron annihilation. CI, such as ANN, was first proposed by Bezdek [37] and it has gained much attention. CI is a set of "nature-inspired" computational algorithms and paradigms to model complex real-world problems for which conventional mathematical modeling can be useless. It also offers us automatic modeling techniques using the measurements of system behavior.

In the last few years, GMDH neural network has become one of the most efficient inductive learning algorithms in the family of computational intelligent techniques for computer-based mathematical modeling of non-linear complex phenomena. GMDH neural network tends to automatically generate a computer program in the form of polynomial equations when it is applied to estimate highly nonlinear complex phenomena. This approach has many privileges: it gives the user knowledge about the system and the way of verification of models constructed by human experts, as well as it saves a lot of time used for manual derivation of model equations.

GMDH models developed in the present work mainly aim to generate the mathematical functions for the prediction and analysis of multiplicity distributions, $P\left(n_{c h}, \sqrt{s}\right)$, of positron-electron hadronic annihilation. According to this model, we have obtained empirical physical equation to calculate and predict the charged-particle multiplicity distribution which takes the form $P\left(n_{c h}, \sqrt{s}\right)$ leading to the calculation of $\overline{\mathrm{n}}$. Once we obtain the equation for $P\left(n_{c h}, \sqrt{s}\right)$, it is easy to obtain the calculation of $\bar{n}$, since $\bar{n}$ is a function of $\sqrt{s}$.

In order to demonstrate the prediction ability and evaluate the generality of GMDH type neural networks, experimental data from several collaboration [12-20] has been used to construct the $P\left(n_{c h}, \sqrt{s}\right)$ model (GMDH) (empirical physical equation). In the present study, experimental data from different Collaborations [12-20] has been used for the model development. The experimental data are divided into training (calibration) set and testing (validation) set (A 10-fold cross-validation method [32] was used to evaluate the estimation error, that is, all the experimental data are randomly divided into 10 folds. 9 of them are used to train the model and the remaining one is used to test it). When the learning stage is finished, the built model is utilized to calculate and predict the output values for data which never been seen during the training stage.

Three different classes of polynomials, namely linear, quadratic, and cubic are utilized, resulting in the proposed model. The functions used in the network are as follows:

$$
\begin{array}{ll}
\text { Quadratic: } 2 \text { variables } & y=q_{1}+q_{2} x_{1}+q_{3} x_{1}^{2}+q_{4} x_{2}+q_{5} x_{2}^{2}+q_{6} x_{1} x_{2} \\
\text { Logarithm: } 1 \text { variable } & y=q_{1}+q_{2} \log \left(x_{1}+q_{3}\right) \\
\text { Exponential: } 1 \text { variable } & y=q_{1}+q_{2} \exp \left(q_{3}\left(x_{1}+q_{4}\right)\right)
\end{array}
$$

Where, $\mathrm{q}_{1}, \mathrm{q}_{2}, \ldots$, are the coefficients of the polynomial functions (estimated the GMDH model during the learning process) and $\mathrm{x}_{1}, \mathrm{x}_{2}$ are the variables. Some network parameters (free parameters) must be specified before the learning process (e.g. the maximum number of layers, polynomial type and order). In this study; the number of layers in the neural network that the model may contain is specified as 20 , and the polynomial order of a variable that a polynomial may contain is specified as 16). Convergence tolerance that will stop the training algorithm from adding a new layer when it reaches the specified value and detects from adding a layer will not improve the model. The number of neurons in each layer of the network is specified as the same number of neurons in the input layer. Network layer connections method controls how neurons in the network are connected together. In this paper, only the type of connection to the previous layer is chosen (This tells DTREG [38] that the inputs to one layer may come only from outputs generated by the next lower layer).

Based on minimum error performance in validation sets the corresponding polynomial representation of the present GMDH model for $P\left(n_{c h}, \sqrt{s}\right)$ is obtained (empirically) as:

$$
\left.P=P\left(n_{c h}, \sqrt{s}\right)=a_{1}+b_{1} e^{\left(c_{1}\left(d_{1}+g_{1}\left(h-I \frac{n_{c h}}{\sqrt{s}}\right) N_{1}\right)+k_{1}\right.}\right),
$$

where,

$$
\begin{aligned}
& \left.N_{1}=a_{2}+b_{2} e^{\left(c_{2}\left(d_{2}+g_{2}\left(h-I \frac{n_{c h}}{\sqrt{s}}\right) N_{2}\right)+k_{2}\right.}\right) \\
& \left.N_{2}=a_{3}+b_{3} e^{\left(c_{3}\left(d_{3}+g_{3}\left(h-I \frac{n_{c h}}{\sqrt{s}}\right) N_{3}\right)+k_{3}\right.}\right) \\
& N_{3}=-0.042996+0.286515 e^{\left(\frac{-\left(\left(N_{4}\right)-0.267503\right)^{2}}{0.040062}\right)} \\
& N_{4}=-0.116698+4.027115 N_{5}-32.31281 N_{5}^{2}+1.599191 N_{6}+4.039759 N_{6}^{2}-16.22014 N_{5} N_{6} \\
& N_{5}=-0.193252-0.027337 \log (\sqrt{s}+37.73184)
\end{aligned}
$$




$$
\begin{aligned}
& N_{6}=-0.008245+11.02105 N_{7} N_{8} \\
& N_{7}=0.168889+2.940838 N_{10}-88.85973 N_{10}^{2}+6.973632 N_{11}+22.60074 N_{11}^{2}+102.1841 N_{10} N_{11} \\
& N_{8}=a_{4}+b_{4}+\left(h-I \frac{n_{c h}}{\sqrt{s}}\right)-c 4\left(h-I \frac{n_{c h}}{\sqrt{s}}\right)^{2} d_{4} N_{9}+g_{4} N_{9}^{2}+k_{4}\left(h-I \frac{n_{c h}}{\sqrt{s}}\right) N_{9} \\
& N_{9}=a_{5}+b_{5}+n_{c h} c_{5} n_{c h}^{2}+d_{5}\left(h-I \frac{n_{c h}}{\sqrt{s}}\right)-g_{4}\left(h-I \frac{n_{c h}}{\sqrt{s}}\right)^{2}+k_{5} n_{c h}\left(h-I \frac{n_{c h}}{\sqrt{s}}\right) \\
& N_{10}=-0.072627-0.002561 \frac{\sqrt{s}}{n_{c h}^{2}} \\
& N_{11}=0.06004+0.002546 n_{c h}-0.000082 n_{c h}^{2} \\
& \mathrm{a}_{1}=-1.296707, \mathrm{~b}_{1}=1.298148, \mathrm{c}_{1}=0.722543, \mathrm{~d}_{1}=-0.000534, \mathrm{~g}_{1}=16.88877, \mathrm{~h}=0.061227, \mathrm{I}=0.004658, \\
& \mathrm{k}_{1}=0.097891, \mathrm{a}_{2}=-1.273491, \mathrm{~b}_{2}=1.274938, \mathrm{c}_{2}=0.735137, \mathrm{~d}_{2}=-0.000559, \mathrm{~g}_{2}=16.89868, \mathrm{k}_{2}=0.098519, \\
& \mathrm{a}_{3}=-1.358375, \mathrm{~b}_{3}=1.359726, \mathrm{c}_{3}=0.692116, \mathrm{~d}_{3}=0.00059, \mathrm{~g}_{3}=16.90984, \mathrm{k}_{3}=0.099141, \\
& \mathrm{a}_{4}=-19.93856, \mathrm{~b}_{4}=684.3732, \mathrm{c}_{4}=-5870.185, \mathrm{~d}_{4}=17.87208, \mathrm{~g}_{4}=7.419097, \mathrm{k}_{4}=308.3482, \\
& \mathrm{a}_{5}=-10.78599, \mathrm{~b}_{5}=0.244696, \mathrm{c}_{5}=-0.000064, \mathrm{~d}_{5}=453.8323, \mathrm{~g}_{5}=4544.448, \mathrm{k}_{5}=4.099441 .
\end{aligned}
$$

The center-of-mass energy dependence of the average charged-particle multiplicity, $\bar{n}$, according to our model GMDH is obtained as:

$$
\bar{n}=A e^{-13}+(B+C\{D+G \log (\sqrt{s}+H)\})-k\{D+G \log (\sqrt{s}+H)\}^{2},
$$

where

$\mathrm{A}=-1.921449, \mathrm{~B}=-0.17183, \mathrm{C}=1.019804, \mathrm{D}=-24.50703, \mathrm{G}=9.628115, \mathrm{H}=9.628115, \mathrm{~K}=0.000532$.

The criteria of root mean square error (MSE) and coefficient of determination $\left(R^{2}\right)$ are used for evaluating the performance of the GMDH models.

\section{RESULTS AND DISCUSSION}

Hadron production in positron-electron annihilation is created from the yield of quark-antiquark pairs which can generate gluons, the exchange particle of the field theory of the strong interactions and QCD. The produced gluon depends on the center of mass energy. Based on the GMDH, the general form empirical equation can be represented by a system of particle description using some type of order polynomial such as, linear, equations, quadratic equations and cubic equations (see equations (8) and (9)).

We investigate the general features of hadronic decays in $e^{+} e^{-} \rightarrow\left(Z_{o} / \gamma\right) \rightarrow q+\bar{q}$ reactions at the highest available centre-of-mass energies. We analyzed the multiplicity distribution of hadronic decays in $e^{+} e^{-} \rightarrow\left(Z_{o} / \gamma\right) \rightarrow q+\bar{q}$ reactions up to the highest available centre-of-mass energies $\sqrt{s}=206 \mathrm{GeV}$. Due to the

physical phenomena correlated with the non-linear characteristics of multiplicity distribution of the $e^{+} e^{-}$hadronic collision, it may be tricky to establish explicit mathematical equation for multiplicity distribution. By adequate operations of mathematical analysis, deduction of highly nonlinear physical equations for multiplicity distribution is of utmost interest.

In this paper, by using group method of data handling (GMDH), we have constructed mathematical functional form for the charged particle multiplicity distribution of $e^{+} e^{-}$hadronic annihilation

The GMDH model is developed by running DTREG software [38]. The simulation results are conducted on a pentium4, with 1.4 GHZ, 2G RAM and Windows XP. With these configurations, the predictive results are obtained in few seconds. A 10-fold cross-validation is used to create and test the model [32].

The proposed GMDH model is tested after training. As shown in Fig. 3, the GMDH technique was able to perfectly model and simulate the charged particles multiplicity distribution, $P\left(n_{c h}, \sqrt{s}\right)$ as well as the center-of-mass energy dependence of the average multiplicity distribution, $\bar{n}=\bar{n}(\sqrt{s})$ of the $e^{+} e^{-}$interactions at high energies. This gives the GMDH the proven ability of wide usage in the modeling of high energy physics.

Figure 3 demonstrates the calculated charged multiplicity distribution at $\sqrt{s}=14,29,34.8,43.6, \ldots ., 206 \mathrm{GeV}$ which have been compared with the corresponding experimental and theoretical ones [21-24]. The comparison between the charged particle multiplicity distribution calculated by the generalized multiplicity distribution (GMD) model for $e^{+} e^{-}$ collisions at $\sqrt{s}=14$, and $206 \mathrm{GeV}$ is shown in Fig. 3. This Figure shows that the proposed model gives a good agreement with the experimental [12-20] and theoretical [21-24] data especially at 183 and $206 \mathrm{GeV}$.

Fig. 4 demonstrates the comparison between the multiplicity distributions for different $\sqrt{s}$. From this figure, we 
notice that the maximum probability of production " $\mathrm{n}_{\mathrm{ch}}$ " charged particles decreases with the increase of $\sqrt{s}$ as well as shifts towards the increase of " $\mathrm{n}_{\mathrm{ch}}$ ". Further, we notice that the width of the distribution is broadened with the increase of $\sqrt{s}$.
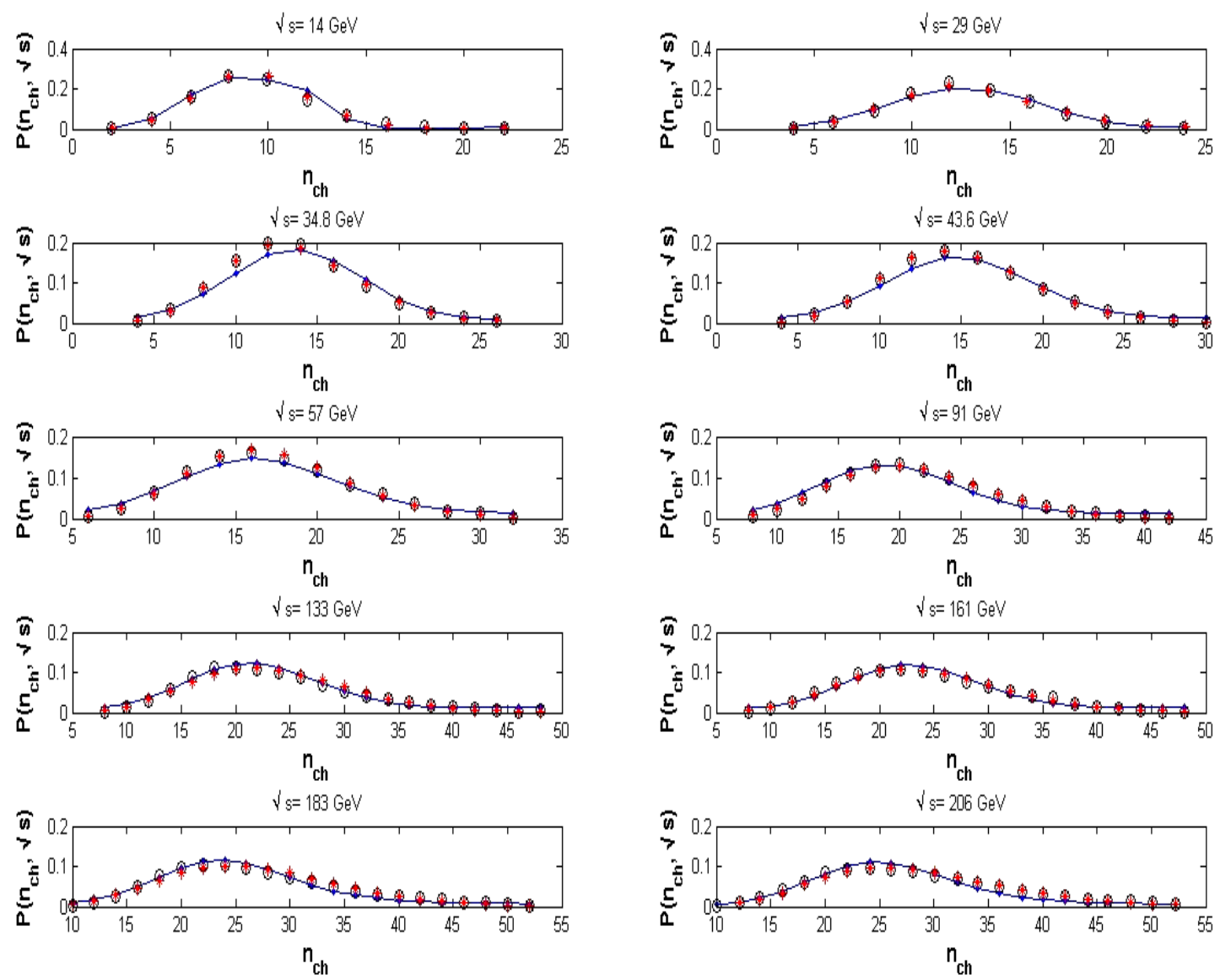

Fig. 3. Demonstrates the calculated charged multiplicity distribution $P\left(n_{c h}, \sqrt{s}\right)$ at $\sqrt{s}=14,29,34.8,43.6, \ldots ., 206 \mathrm{GeV}$ those have been compared with experimental [12-20] and theoretical ones "GMD Model" [21-24]. (-) GMDH model, experimental data (o) and $(*)$ theoretical ones.

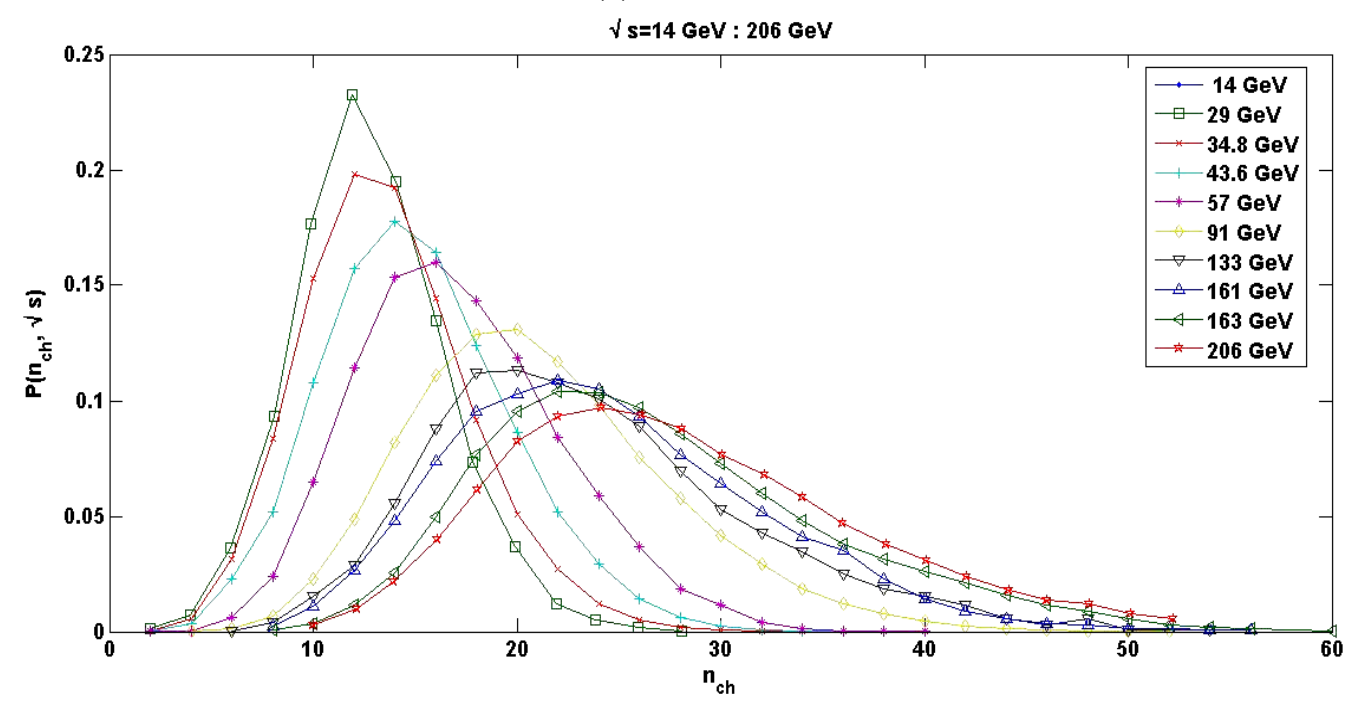

Fig. 4. The calculated charged multiplicity distribution $P\left(n_{c h}, \sqrt{s}\right)$ at $\sqrt{s}=14,29,34.8,43.6, \ldots ., 206 \mathrm{GeV}$.

Based on the obtained equations (eqs. (8) and (9)), the values of energy dependence of the average charged multiplicity in $e^{+} e^{-}$collision for $\sqrt{s}$ ranging from 14 to $206 \mathrm{GeV}$ are calculated and compared with corresponding experimental and theoretical results as shown in Fig. 5. Also, From Fig.5 we notice that, $\bar{n}$, increase with the increase 
of $\sqrt{s}$ which shows the same trend as the experiment.

The average multiplicity of charged particles at a hadronic energy at the highest centre of mass energies in $e^{+} e^{-}$interactions, up to $206 \mathrm{GeV}$ has been calculated to be $\bar{n}$. When compared to other models, the value calculated is consistent with the evolution predicted by GMD method [21-24]. When $\sqrt{s}=206 \mathrm{GeV} \bar{n}=27.5$, and so on for the lower energies. The results prove that the proposed GMDH model has impressively learned well the nonlinear behavior of the charged multiplicity distribution and the average charged multiplicity in $e^{+} e^{-}$collision.

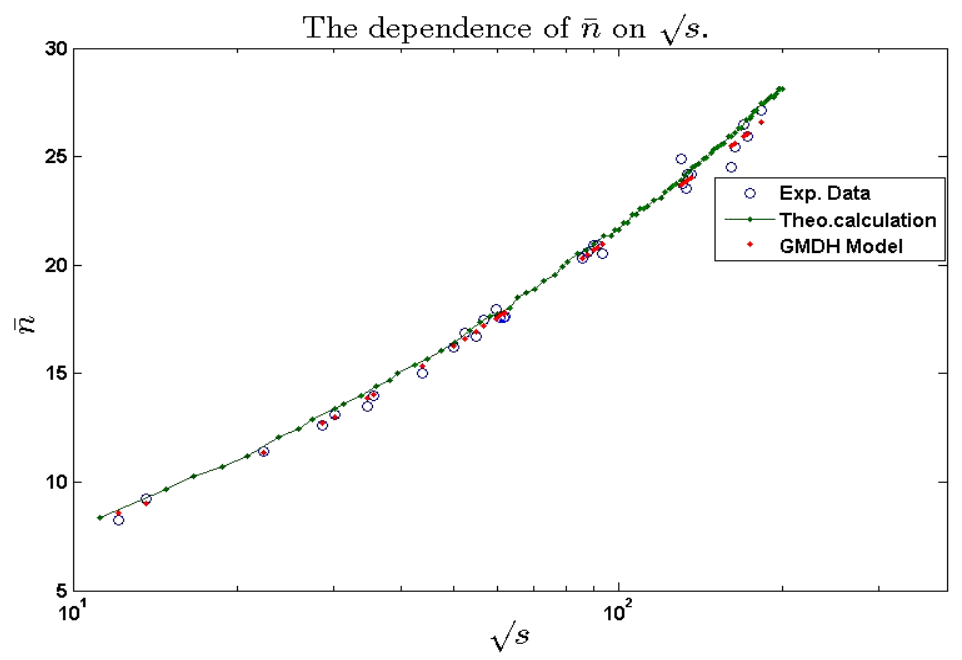

Fig. 5. The comparison between the experimental and theoretical values of the energy dependence of the average charged multiplicity in $e^{+} e^{-}$annihilation: (o) experimental data, ( $\bullet$ GMDH model, and (- $\bullet$ ) theoretical ones [21-24].

\section{CONCLUSIONS}

We have obtained an empirical physical equation for the description of the charged particles multiplicity distribution in hadronic positron-electron annihilation based on GMDH approach. We have used the obtained equation to calculate and predict $P\left(n_{c h}, \sqrt{s}\right)$ through the energy range $14 \mathrm{GeV}$ to $206 \mathrm{GeV}$. The average multiplicity, $\bar{n}$, is calculated using our empirical physical equation. The comparison between our results and the experimental and theoretical ones explores a good agreement. Based on our GMDH model, we present an empirical equation corresponding to physical phenomenon in a mathematical form, we are still faced with the challenge of justifying and giving words to their meaning. These models define input-output relations based on experimental data and use mathematical and statistical concepts to link input to model the output. Our results are in a good agreement with the experimental and theoretical ones. These results confirm the reliability of our models. Scientists may use such approaches to focus on interesting phenomena more rapidly and to interpret their meaning. This represents one of the key challenges for computational Intelligence techniques in high energy physics modeling.

\section{ACKNOWLEDGEMENTS}

The authors are very grateful to the Editor-in-Chief and the anonymous referees for their helpful comments and for the kind suggestions that improved the manuscript seriously.

\section{REFERENCES}

1. Zheng H.W., Perez P., Auchincloss P., Blanis D., Bodek A., Budd H., Kim Y.K. Charged-particle multiplicities in e+eannihilations at $\sqrt{S}=50-61.4 \mathrm{GeV} / /$ Phys. Rev. D. $-1990 .-$ Vol. $42 .-$ P. 737.

2. Ghaffary T. Charged Particles Multiplicity and Scaling Violation of Fragmentation Functions in Electron-Positron Annihilation // Advances in High Energy Physics. - 2016. - Vol. 2016. - Article ID 4506809. - P.8; doi:10.1155/2016/4506809.

3. Bzdak A. Universality of multiplicity distribution in proton-proton and electron-positron collisions. - $2015 / /$ ePrint: arXiv:1507.01608 [hep-ph].

4. Biebel O. Experimental tests of the strong interaction and its energy dependence in electron-positron annihilation // Phys. Rev. $-2001 .-$ Vol. 340. - P.165-289.

5. Derrick M., Gan K.K., Kooijman P., Loos J.S., Musgrave B., Price L.E., Wood D.E. Study of quark fragmentation in e+eannihilation at $\sqrt{S}=29 \mathrm{GeV}$ : Charged-particle multiplicity and single-particle rapidity distributions // Phys. Rev. D. - 1986. Vol. 34. - P.3304-3320.

6. Wyatt T. High-energy colliders and the rise of the standard model // Nature. - 2007. - Vol.448. - P. 274-280.

7. Ackerstaff K., Alexander G., Allison J., Altekamp N., Ametewee K., Anderson K.J., Azuelos G. QCD studies with e+ eannihilation data at $\sqrt{S}=161 \mathrm{GeV} / /$ Z. Phys. C Part. Fields. -1997 . - Vol. 75. - P.193-207. 
8. Beggio P.C., Luna E.G.S. Cross sections, multiplicity and moment distributions at the LHC // Nucl. Phys. A,. - 2014. Vol. 929. - P. 230-245.

9. Acton P.D., Alexander G., Allison J., Allport P.P., Anderson K.J., Arcelli S., Bahan G.A. A study of charged particle multiplicities in hadronic decays of the Z0 // Z. Phys. C Part. Fields. - 1992. - Vol. 53. - P. 539-554.

10. Abreu P., Adam W., Adami F., Adye T., Alexeev G.D., Allen P., Anassontzis E. Charged particle multiplicity distributions in Z0 hadronic decays // Z. Phys. C Part. Fields. - 1991. - Vol. 50. - P.185-194.

11. Dremin I.M. QCD and models on multiplicities in $\mathrm{e}+\mathrm{e}-$ and interactions // Phys Atom Nucl+. - 2005. - Vol. 68. - P. 758-770.

12. ALEPH Collaboration, DELPHI collaboration, L3 Collaboration, OPAL Collaboration, \& LEP Electroweak Working Group. Electroweak measurements in electron-positron collisions at W-boson-pair energies at LEP // Phys. Rept. - 2013. - Vol. 532. P. 119-244.

13. Braunschweig W., Gerhards R., Kirschfink F.J., Martyn H.U., Fischer H.M., Hartmann H., Foster B. Charged multiplicity distributions and correlations in $\mathrm{e}+\mathrm{e}-$ annihilation at PETRA energies // Z. Phys. C Part. Fields. C45. - 1989. - Vol. 45. P. 193-208.

14. Achard P., Adriani O., Aguilar-Benitez M., Alcaraz J., Alemanni G., Allaby J., Anselmo F. Studies of hadronic event structure in e+e- annihilation from 30 to $209 \mathrm{GeV}$ with the L3 detector // Phys. Rept. - 2004. - Vol. 399. - P. 71-174.

15. Alexander G., Allison J., Altekamp N., Ametewee K., Anderson K.J., Anderson S., Ball A.H. QCD studies with e+eannihilation data at 130 and $136 \mathrm{GeV} / /$ Z. Phys. C Part. Fields. - 1996. - Vol. 72. - P. 191-206.

16. Radchenko N.V. About agreement of PYTHIA and the experimental results in e+e- annihilation to hadrons. - 2007. e-print: arXiv:0706.3453 [hep-ph].

17. Abbiendi G. QCD studies with annihilation data at $\sqrt{S}=172-189 \mathrm{GeV} / /$ The Eur. Phys. J. C. - 2000. - Vol. 16. P. 185-210.

18. Abreu P., Adam W., Adye T., Agasi E., Ajinenko I., Aleksan R., Amaldi U. Charged particle multiplicity in e+e- interactions at $\sqrt{S}=130 \mathrm{GeV} / /$ Phys. Lett. B. $-1996 .-$ Vol. 372. - P.172-180.

19. Heisenberg W. Production of meson showers // Nature. - 1949. - Vol. 164. - No. 4158. - P. 65-65.

20. Fermi E. High energy nuclear events // Prog. Theor. Phys. - 1950. - Vol. 5. - P. 570-583.

21. Dewanto A., Chan A.H., Oh C.H., Chen R., Sitaram K. Lee-Yang circle analysis of e+e- and generalized multiplicity distribution // Eur. Phys. J. C. - 2008. - Vol. 57. - P. 515-523.

22. Chew C.K., Kiang D., Zhou H. A generalized non-scaling multiplicity distribution // Phys Lett. B. - 1987. - Vol. 186. - P. 411415.

23. Chan A.H., Chew C.K. e+e- multiplicity distribution from branching process // Z. Phys. C Part. Fields. - 1992. - Vol. 55. P. 503-508.

24. Zborovský I. Multiplicity distributions in proton-(anti) proton and electron-positron collisions with parton recombination. 2011. e-print arXiv:1106.4697 [hep-ph].

25. Link J.M., Yager P.M., Anjos J.C., Bediaga I., Castromonte C., Göbel C., Pepe I.M. Application of genetic programming to high energy physics event selection // Nucl. Instr. Meth. Phys. Res. A. - 2005. - Vol. 55. - P. 504-527.

26. Schmidt M., Lipson H. Distilling free-form natural laws from experimental data // Science. - 2009. - Vol. 324. - P. 81-85.

27. Akkoyun S., Bayram T. Estimations of fission barrier heights for Ra, Ac, Rf and Db nuclei by neural networks // Int. J. Mod. Phys. E. - 2014. - Vol. 23. - P. 1450064.

28. Akkoyun S., Bayram T., Turker T. Estimations of beta-decay energies through the nuclidic chart by using neural network // Radiat. Phys. Chem. - 2014. - Vol. 96. - P. 186-189.

29. Bhat P., Lonnblad L., Meier K., Sugano K. Using neural networks to identify jets in hadron-hadron collisions. / Research directions for the decade: Proceedings of the 1990 summer study on high energy physics. -1992.

30. Baldi Pierre, Cranmer K., Faucett T., Sadowski P., Whiteson D. Parameterized neural networks for high-energy physics // Eur. Phys. J. C. - 2016. - Vol. 76:235.

31. Alves A. Stacking machine learning classifiers to identify Higgs bosons at the LHC. (2016). e-print arXiv:1612.07725 [hep-ph].

32. Haykin S.S. Neural networks and learning machines (Vol. 3). - 2009. Uppr Saddle River, NJ, USA. Pearson.

33. Ivakhnenko A.G. Polynomial theory of complex systems // IEEE Trans. on Systems, Man and Cybernetics SMC. - 1971. - Vol. 1. - P. 364-378.

34. Madala H.R., Ivakhnenko A.G. Inductive learning algorithms for complex systems modeling // Boca Raton. - 1994. - 368p. CrC Press.

35. Mueller J.A., Ivachnenko A.G., Lemke F. GMDH algorithms for complex systems modeling // Math. Comput. Model. Dyn. Syst. - 1998. - Vol. 4. - P. 275-316.

36. Farlow S.J. Self-organizing methods in modeling: GMDH type algorithms. - 1984. - Vol. 54. - CrC Press.

37. Bezdek J.C. On the relationship between neural networks, pattern recognition and intelligence // Int J Approx Reason. - 1992. Vol. 6. - P. 85-107.

38. Sherrod P.H. DTREG Predictive Modeling Software. - 2008. - Manual for software available online: www.dtreg.com. 